\title{
Success rate of bedside PICC placement and characteristics of its tip position
}

HaengJin OHE ${ }^{1}$, Soo mi Son², Joung Hee Kim², Jang Yong KIM²,

${ }^{1}$ Vascular and Transplant Surgery School of Medicine Inje University Seoul Paik Hospital, ${ }^{2}$ Vascular and Transplant Surgery School of Medicine The Catholic University of Korea Seoul St. Mary's Hospital

\section{Introduction}

This study was conducted to evaluate the incidence and risk factors of tip malposition after performing bedside PICC.

\section{Methods}

This is a retrospective study from prospectively registered database from patients who underwent PICC placement from Jan 2013 to September 2014. Success rate of bedside PICC, clinical characteristics of tip position after bedside PICC placement and risk factors of tip malposition was analyzed.

\section{Results}

Overall success rate of venous access in bedside PICC placement was $97.1 \%(1289 / 1327)$. The success rate of venous access was $98.6 \%(868 / 880)$ with ultrasound guidance and $97.1 \%$ (434/447) with blind approach, respectively. The proper tip position was ascertained in 1231 (94.5\%) after first attempt.
The repositioning of PICC was tried in 71 patients. 47 PICCs were repositioned at the bedside and 2 PICCs were repositioned under fluoroscopic guide. 3 PICCs were naturally moved to the desired position without intervention. 8 PICCs were used as a midline catheter and 11 PICC catheters were removed. The risk factors of tip malposition were higher age $(p<0.001)$, catheter type $(p<0.001)$ and presence of cancer $(p=0.037)$ or lung disease $(p<0.001)$.

\section{Conclusion}

Bedside PICC is effective and safe methods for CVC with high success rate. To improve success rate of bedside PICC placement, ultrasound guidance is recommended. Old age, cancer and pulmonary disease were significant risk factors of catheter tip malposition. 